\title{
Changes in Erythrocyte Properties during the First Hours of Life: Electron Spin Resonance of Reacting Sulfydryl Groups
}

\author{
RODOLFO BRACCI, GIACOMO MARTINI, GIUSEPPE BUONOCORE, BEATRICE TALLURI, \\ SILVIA BERNI, MARIA FRANCESCA OTTAVIANI, MARIA PIA PICCHI, AND ADRIANA CASINI \\ Division of Neonatology [R.B., G.B., B.T., S.B.] and Department of Chemistry [M.P.P., A.C.], University of \\ Siena, Siena; and Department of Chemistry [G.M., M.F.O.], University of Florence, Florence, Italy
}

\begin{abstract}
In an attempt to clarify the mechanism by which the red cells (RBC) of newborn infants are protected against oxidative agents, electron spin resonance (ESR) assays were carried out using the nitroxide radical 4maleimide-2,2,6,6-tetramethylpiperidinyl-1-oxyl (Mal-6), a sulfydryl-reacting agent. The ESR assays were performed in 24 samples of cord blood, 20 samples of blood from 4day-old infants, and eight samples of 8-h-old infants. The analyses were carried out on whole blood and washed erythrocytes were resuspended in buffered saline. The same experiments were performed in 10 blood samples from healthy adults as controls. Whole blood, before and after removing the buffy coat, and cell-free plasma were also examined by ESR assay. Cell-free plasma and buffy coats proved not to be appreciably involved in the Mal-6 behavior. The data of the ESR spectroscopy demonstrated a significantly slower reaction rate in the samples of cord blood and in blood of 8-h-old infants, compared to that of 4-day-old infants and adults. No significant differences in Mal-6 behavior could be detected between cord blood and 4-day-old infant blood in the results of ESR assays performed in washed red cells. Chemical determination of RBC-reacting sulfydryl groups and the assays of glutathione also demonstrated the absence of differences between cord blood and blood of 4-day-old infants. The results of our investigation suggest that the RBC-sulfydryl-reacting groups are less involved in the detoxification of oxidative agents during the first hours of life than in the following days. This peculiarity of RBC of younger infants appears to be due, to a considerable extent, to the modulation by plasma factors of the interactions between Mal-6 and RBCreacting sulfydryl groups. Therefore, the changes in plasma components occurring during the first hours of life appear to modify the interactions between the RBC and the oxidative agents. (Pediatr Res 24: 391-395, 1988)
\end{abstract}

Abbreviations

RBC, red blood cells

ESR, electron spin resonance

$-\mathrm{SH}$, sulfydryl-reacting groups

Mal-6, 4-maleimide-2,2,6,6-tetramethylpiperidinyl-1-oxyl

Htc, hematocrit

GSH, glutathione

Received January 19, 1987; accepted May 24, 1988

Correspondence and reprint requests Dr. Rodolfo Bracci, Division of Neonatology, University of Siena, 46 Via Paolo Mascagni, 53100 Siena, Italy.

Supported in part by Grant C. 85.00466 from C.N.R. (Consiglio Nazionale delle Ricerche).
Increased susceptibility to oxidative damage is a well-known characteristic of neonatal erythrocytes $(1,2)$. It is responsible for increased formation of Heinz bodies during the incubation of $\mathrm{RBC}$ with oxidative agents and it probably contributes to the shorter life span and membrane abnormalities that have been observed in neonatal erythrocytes.

The causes of this increased susceptibility are still unclear, although the roles played by vitamin E deficiency, and by low $\mathrm{RBC}$ oxygen scavenger enzyme activities have been demonstrated (2-4).

Inasmuch as - $\mathrm{SH}$ groups particularly account for the protection from oxidative injury of the cell (5), the present investigation was undertaken in the attempt to ascertain if this important factor could be involved in the peculiar susceptibility of infant $\mathrm{RBC}$ to oxidative hemolysis.

The ESR technique of spin labeling was used because of its widespread use in the study of properties of the erythrocyte membrane (6). In particular, the spin label Mal-6 was reported to react specifically with -SH groups of proteins, in particular spectrin, band 3 , band 2.1 , and other high molecular weight proteins (7). Spectra with two different correlation times for the motion were usually observed in erythrocyte ghost studies (810 ). They were attributed to weakly bound (more mobile) and to strongly bound (more immobilized) spin labels. Several membrane abnormalities were detected from the weak/strong ratio (7). As with other nitroxides, Mal-6 may be destroyed (reduced) with loss of paramagnetism that can be detected as a decrease in the ESR signal intensity, particularly in the first stages of Mal-6 interaction with some of the biological components in intact systems $(11,12)$. A common source of decreased ESR intensity resides in the one-electron reduction by -SH groups (12). In this study, we found that in whole erythrocytes the reduction reaction was slow enough to be used for a kinetic analysis of the defense against oxidative damage due to -SH groups or other reducing components. Therefore, the Mal-6 ESR assays were carried out in intact $\mathrm{RBC}$ with the purpose of obtaining information about the true conditions of the cells during the first days of life.

\section{SUBJECTS}

Male and female full-term newborn infants were examined. Weights ranged from 2900 to $4200 \mathrm{~g}$. All neonates were free from asphyxia, severe jaundice, hypoglycemia, and other pathological conditions. Apgar scores at $1 \mathrm{~min}$ was $\geqslant 8$. Labor and delivery were carefully studied to exclude infants with perinatal abnormalities or those whose mothers were given drugs during the perinatal period. All newborns were given glucose and water during the first hours of life and were then breast-fed. A few of them were given formula during the first days of life. Healthy adult subjects were examined as controls. 


\section{MATERIALS AND METHODS}

Each subject was tested for $\mathrm{Htc}, \mathrm{Hb}$ concentration, and GSH RBC content. All assays were carried out in the cord blood and were repeated in venous blood taken from the same infants $8 \mathrm{~h}$ or 4 days after birth. No infant had more than one venipuncture. A total of $2 \mathrm{ml}$ of blood was mixed with heparin $(5 \mathrm{U} / \mathrm{ml})$. For the experiments performed with washed $\mathrm{RBC}$, the whole blood was centrifuged at $1500 \times g$ for $10 \mathrm{~min}$ at $36.5^{\circ} \mathrm{C}$ and $\mathrm{RBC}$ free from leukocytes and platelets were resuspended in buffered saline solution at $\mathrm{pH}$ 7.4. Experiments with washed $\mathrm{RBC}$ were carried out after washing the erythrocytes three times with isotonic phosphate-buffered saline $\left(0.03 \mathrm{M} \mathrm{KH}_{2} \mathrm{PO}_{4}+\mathrm{Na}_{2} \mathrm{HPO}_{4}, 290\right.$ mosm $/ \mathrm{kg}$; $\mathrm{pH} 7.42$ at $36.5^{\circ} \mathrm{C}$. GSH was assayed by the method of Beutler (13).

Determinations of total sulfydryl groups were carried out in 15 newborns tested twice, in cord blood erythrocytes, and in erythrocytes of 4-day-old infants by the method of Sedlak and Lindsay (14).

Blood of infants whose hematological picture was determined as reported above, was used for ESR spectra. In each case, cord blood and blood drawn at the 8th $h$ of life or the 4th day of life were examined. Cord blood and blood from 8-h-old infants were examined in eight cases, whereas cord blood and blood from 4 day-old infants were examined in 24 and 20 subjects, respectively. ESR assays were carried out in whole blood and in washed erythrocytes resuspended in buffered saline at $\mathrm{pH}$ 7.4. Hb concentration was determined in the whole blood and in the final RBC suspension. RBC free of plasma were also tested for ESR spectra. Three experiments were carried out by repeating the ESR assays in whole blood in the same subjects, before and after removing the buffy coat. Whole blood and washed erythrocytes from 10 adult subjects resuspended in buffered saline were assayed as controls.

Mal-6 was purchased from Syva (Palo Alto, CA) and used without further purification. A GSH/Mal-6 ratio of $1 / 1$ was used for each sample by adding the appropriate amount of a $2 \times 10^{-3}$ $\mathrm{mol} /$ liter water solution of the spin label to samples of whole

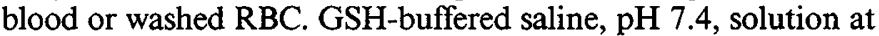
a concentration of $10^{-3} \mathrm{~mol} /$ liter was also prepared as a control of the signal decrease due only to -SH groups.

The ESR spectra were recorded on a Bruker 200tt spectrometer operating in the $\mathrm{x}$-band, using sealed quartz capillaries. Instrumental settings were regulated to avoid overmodulation and power saturation of the spectral lines. Temperature was controlled by a Bruker ST100/700 variable temperature accessory and was kept at $25 \pm 1^{\circ} \mathrm{C}$. Each ESR measurement was carried out no more than $10 \mathrm{~h}$ after blood sampling.

Kinetic analysis was carried out by following the decrease in the height of the central component of the hyperfine pattern of the ESR spectrum of Mal- 6 as a function of time. The starting point was always $3 \mathrm{~min}$ after the addition of the spin label and the kinetics were followed instrumentally for at least $50 \mathrm{~min}$. ESR height, after correction for spectral gain, was taken as line intensity, because no variation was observed in line width as a function of time. Accuracy was $\pm 3 \%$ with respect to the arbitrary value of 100 taken as the starting point.

The preliminary experiments demonstrated that the decrease in intensity of the ESR signal occurred in about the first $60 \mathrm{~min}$ after addition of the spin probe, after which the signal intensity stabilized. ESR spectra registered after overnight incubation at $4^{\circ} \mathrm{C}$ consisted in an overlapping of two absorptions (Fig. 4) that were generally attributed to Mal-6 bound to mobile -SH groups (peaks marked with W) and to Mal-6 bound to partially immobilized -SH groups (peaks marked with $\mathrm{S}$ ) of the membrane proteins, respectively $(7-10)$. This indicates that in the very early stages of the interaction between spin label and biological components, only a fraction of the radical is reduced, whereas the remaining fraction undergoes nonreductive reaction and is covalently bounded to membrane -SH. In this study we decided not to use the $\mathrm{W} / \mathrm{S}$ ratio criterion (as done in several previously

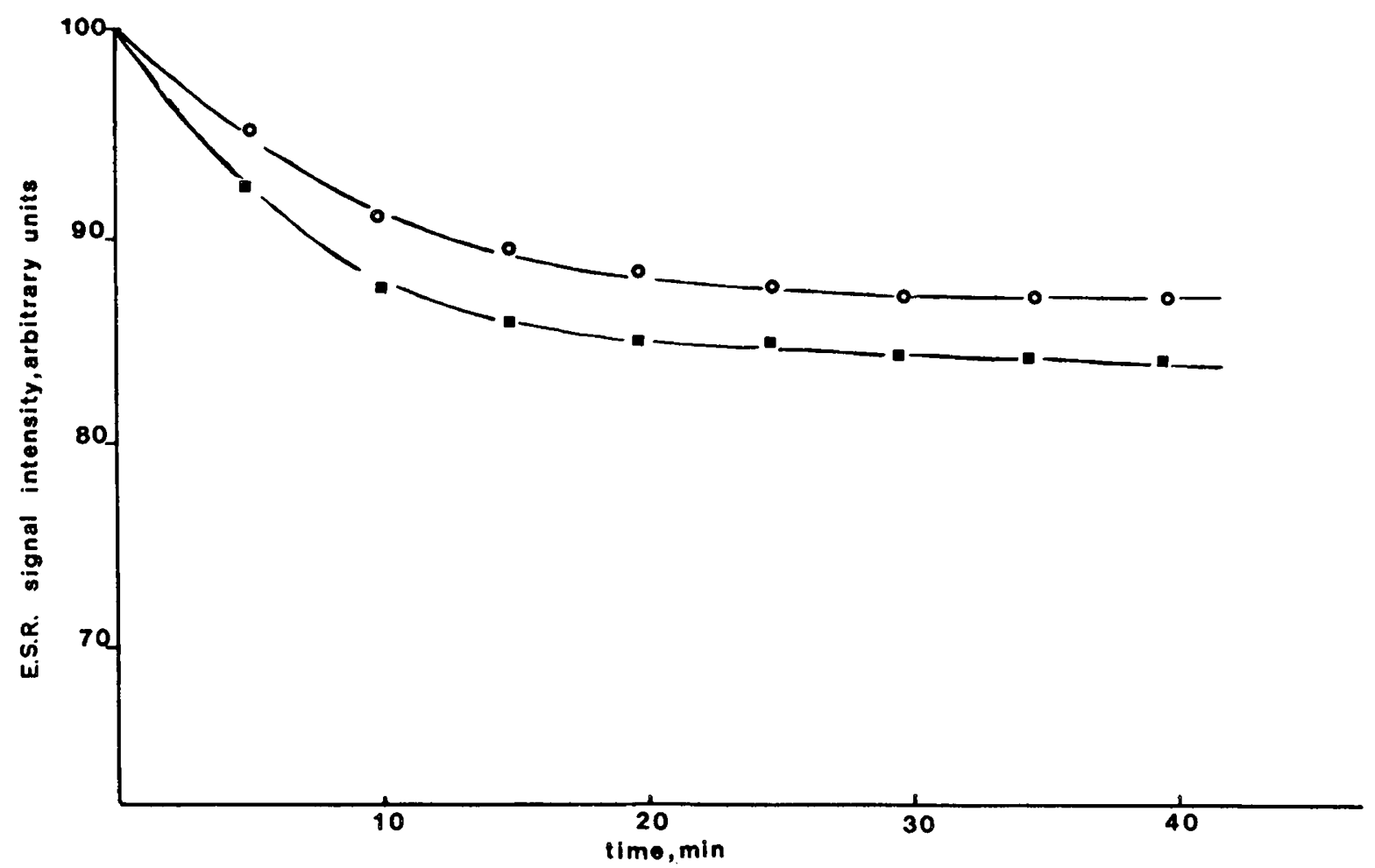

Fig. 1. Intensity (arbitrary units) of the central hyperfine line $\left(m_{1}=0\right)$ of the ESR spectrum of the maleimide spin label Mal- 6 as a function of time: (circles) Mal-6 $10^{-3} \mathrm{~mol} / \mathrm{liter}$ aqueous solution in the presence of GSH $5 \times 10^{-4} \mathrm{~mol} / \mathrm{liter}$ aqueous solution; (squares) Mal-6 as above; GSH $10^{-3} \mathrm{~mol} / \mathrm{liter}$. 
published reports), because the evaluation of this parameter in living cells, as used here, seemed to be rather uncertain.

The statistical analysis of the data was performed by Student's $t$ test for paired data to compare the values found in cord blood and those found in 8-h- and in 4-day-old infants. Student's $t$ test for grouped data was used to compare other values detected.

\section{RESULTS}

$\mathrm{Hb}(15.2 \pm 0.3$ versus $16.5 \pm 0.4 \mathrm{~g} / \mathrm{dl})$ and $\mathrm{Htc}(50.2 \pm 3.0$ versus $58.5 \pm 6.3 \%$ ) increased slightly from birth to the 4 th day of life.

No significant differences were observed between the levels of

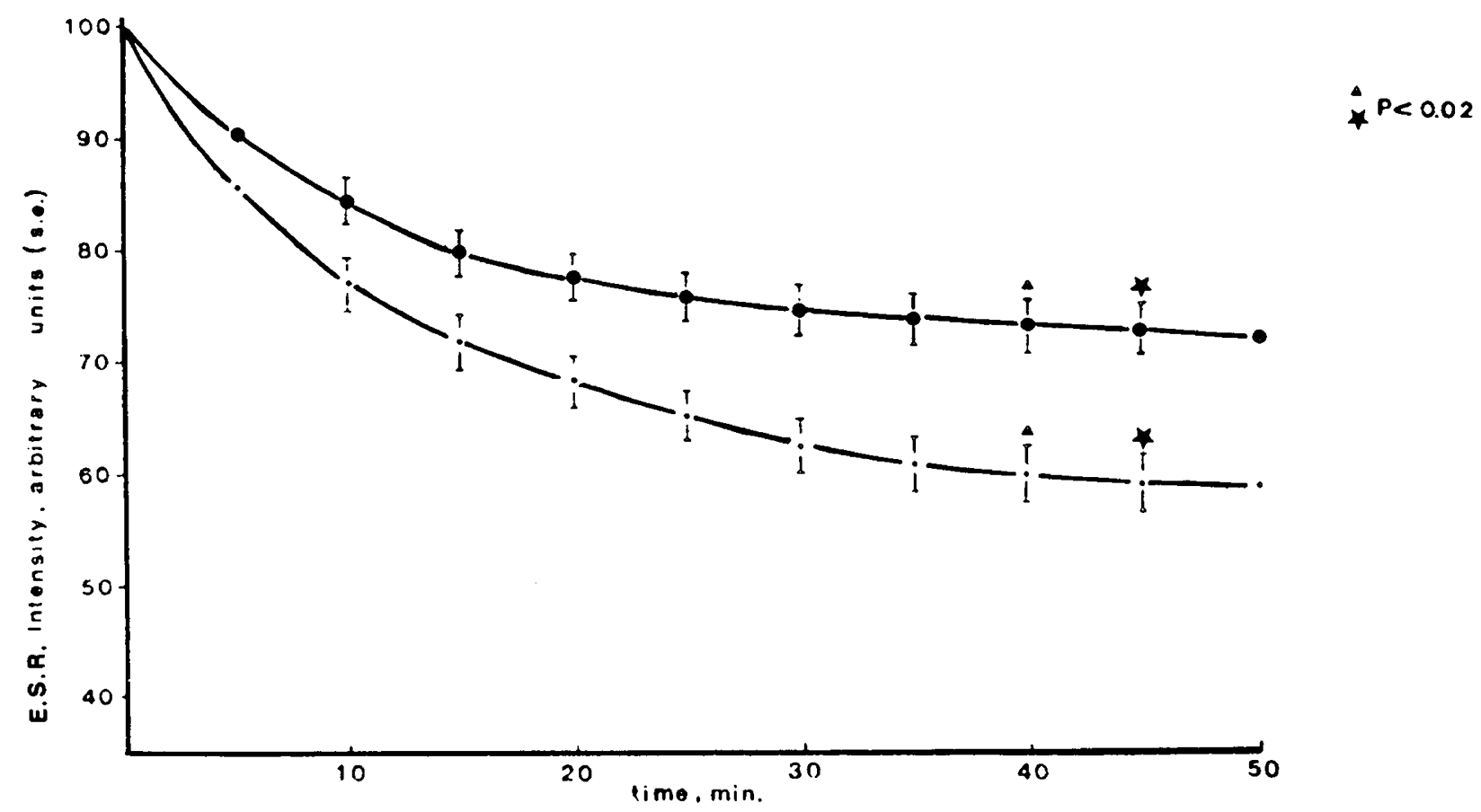

Fig. 2. ESR signal intensity (arbitrary units) of the central hyperfine line of the Mal-6 spectrum as a function of time in cord (large circles) and in fourth day blood (small circles). Mal-6/GSH ratio $=1$.

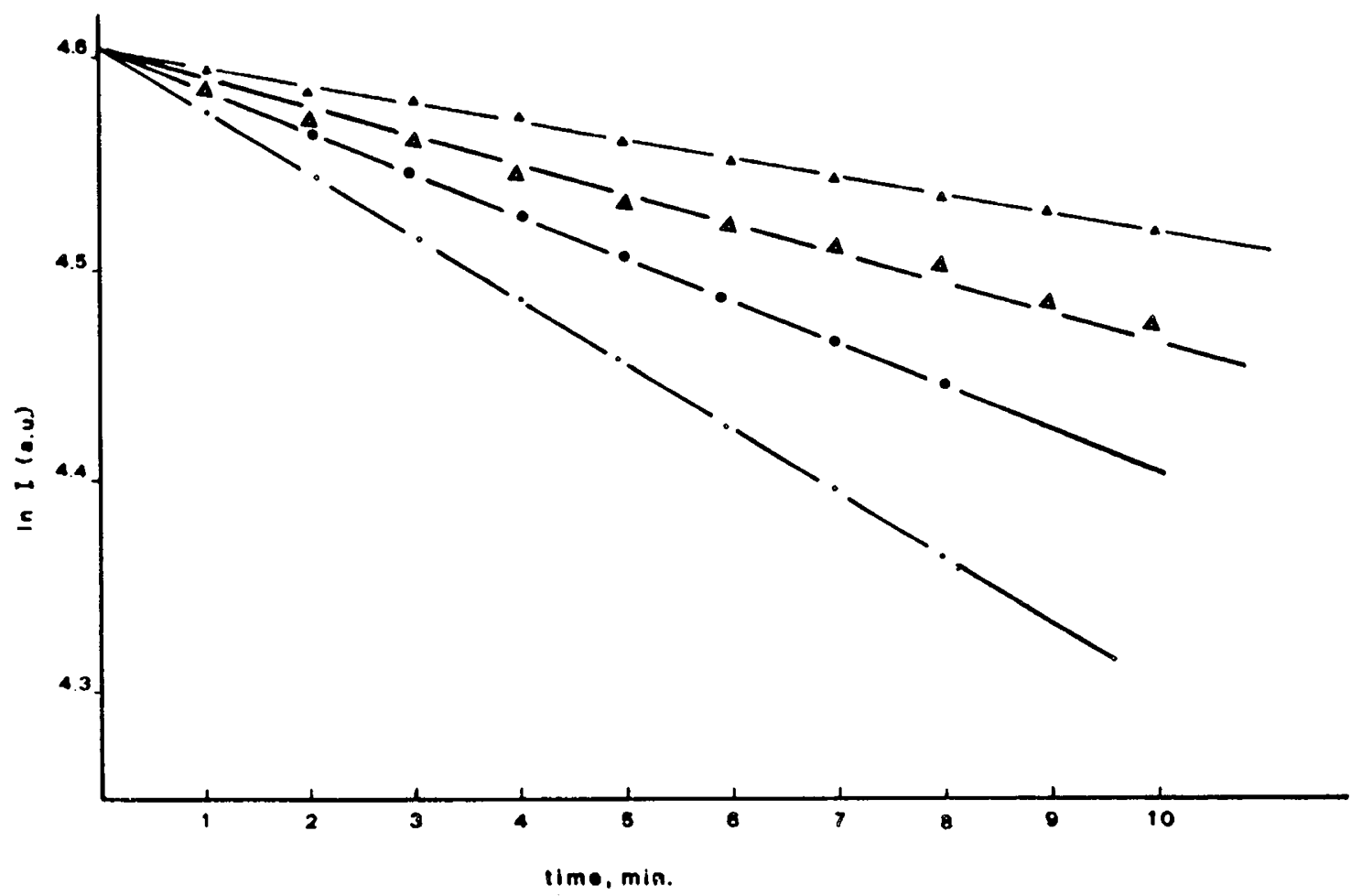

Fig. 3. ESR intensity in a logarithm scale of the central hyperfine line of the Mal-6 spectrum as a function of time in the early stages of the nitroxide radical reduction: in buffered GSH solution with Mal-6/GSH ratio 1/1 (large triangles) and 2/1 (small triangles); in cord (large circles) and fourth day blood (small circles) with Mal-6/GSH ratio $1 / 1$. 
GSH $(8.4 \pm 0.4$ versus $7.9 \pm 0.4 \mu \mathrm{mol} / \mathrm{g} \mathrm{Hb})$ in the first and second samples. No significant differences were observed in the comparison between the results of chemically assayed -SH groups in cord blood erythrocytes and in those of 4-day-old infants $(98.39 \pm 4.02$ versus $105.44 \pm 5.95 \mathrm{nmol} / \mathrm{mg}$ protein; $\bar{x} \pm \mathrm{SE})$.

Figure 1 shows the signal decrease of the $\mathrm{m}_{1}=0$ component of the Mal- 6 spectrum at two different Mal-6/GSH ratios, and Figure 2 shows the intensity of the Mal- 6 spectrum as a function of time in blood of newborns taken at birth and on the 4th day. The signal decrease.in blood taken after $8 \mathrm{~h}$ was the same, within the limit of experimental errors, as that observed in cord blood, and is not reported in Figure 2.

The comparison of the rates of intensity decrease of the ESR spectrum of Mal-6 in the GSH solutions and in newborn blood clearly indicated that a relatively high fraction of the radical reduction could be attributed to free -SH groups. In vivo, other reducing agents could account for the rest of the signal decay.

Figure 3 reports the logarithmic trend of the intensity decrease in GSH and whole blood samples. The good linearity of $1 \mathrm{nI}$ against time confirmed that the Mal- 6 reduction was a first order reaction with rate constants as follows: Mal-6/GSH 2/1, $K=9.0$ $( \pm 0.5) \times 10^{-3} \mathrm{~min}^{-1}$; Mal-6/GSH $1 / 1, K=13.0( \pm 2.0) \times 10^{-3}$ $\mathrm{min}^{-1}$; Mal-6/GSH Cord blood, $K=20.0( \pm 4.0) \times 10^{-3} \mathrm{~min}^{-1}$; Mal-6/4th day blood, $K=29.0( \pm 4.0) \times 10^{-3} \mathrm{~min}^{-1}$.

The comparison of the intensities and the rate constants demonstrated a significantly lower rate of signal decay in cord blood and in blood drawn after $8 \mathrm{~h}$ of life compared with samples taken after 4 days. However, the comparison between washed erythrocytes from cord blood and blood of 4-day-old infants demonstrated no differences in signal decay rate (Fig. 5). This decay rate is significantly lower than that obtained from whole cord blood. Washed erythrocytes from adult subjects also demonstrated a similarly low decay rate (Fig. 5). No detectable signal decay was found in the ESR spectra from cell-free plasma. This last finding demonstrates that plasma components alone do not interfere in the ESR spectra. Inasmuch as no significant differences between intact and buffy coat-free whole blood have been detected, the role of platelets and leukocytes in Mal- 6 reaction appears to be negligible. Signal decay of Mal-6 in whole blood and in washed erythrocytes in adult controls showed the same trend as that found in 4-day-old newborns (Fig. 5). Therefore, a comparison between the behavior of ESR intensity of Mal- 6 in newborn blood and in adult blood demonstrated that the abnormal pattern was that found in cord blood.

\section{DISCUSSION}

During the first days of life a moderate loss of RBC takes place even in healthy, full-term newborns; this seems to account for some cases of hyperbilirubinemia of unknown etiology (15). It is likely that this increased hemolysis results from the incapacity of some erythrocytes to adapt to the extrauterine environment because of their well-proved increased susceptibility to oxidative hemolysis (1).

Although several changes in erythrocyte properties, such as modification in the fatty acid pattern (16 increase in glycolytic)

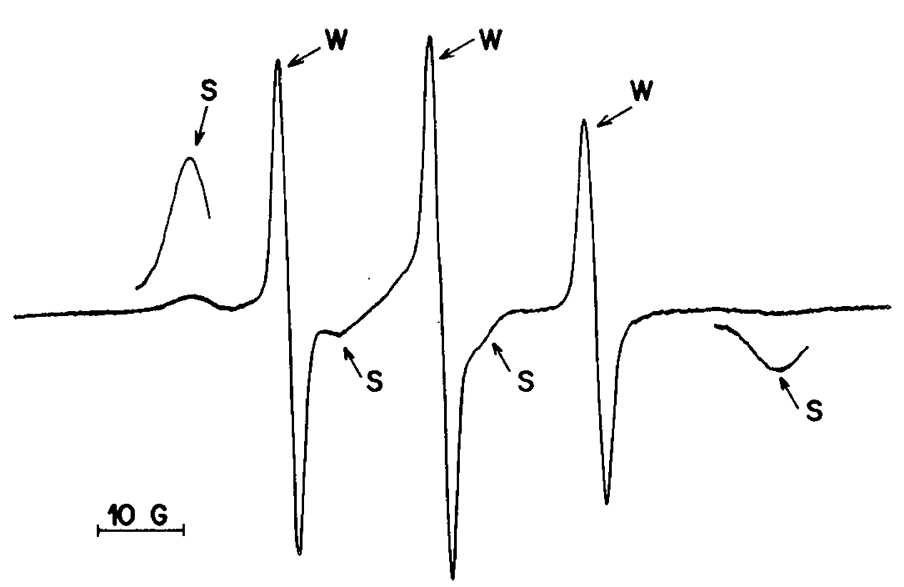

Fig. 4. ESR spectrum of Mal-6 in newborn blood after incubation at $4^{\circ} \mathrm{C}$ overnight. The peaks labeled with $\mathrm{W}$ are due to the absorption of Mal-6 bonded to mobile -SH group whereas S peaks are due to the absorption of Mal- 6 bonded to partially immobilized -SH group.

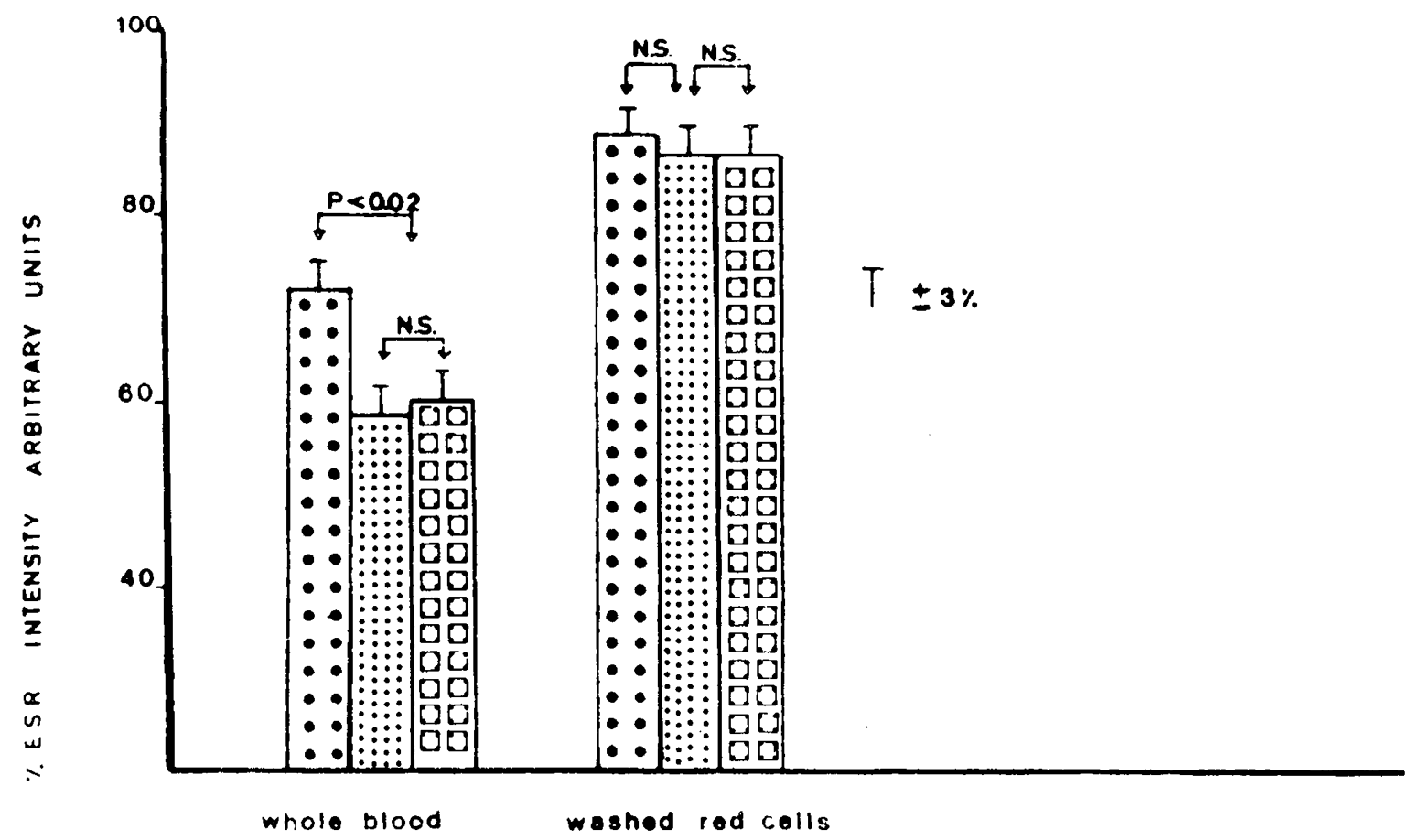

Fig. 5. Residual intensity after 50 min of the ESR spectra of Mal-6 in whole blood and washed RBC of cord (large circles), 4-day-old newborns (small circles), and adult blood (squares). 
intermediates (17), and changes of RBC population (18) have been reported to occur shortly after birth, other factors possibly involved in the protection of the cell from oxidative hemolysis, such as the characteristics of their membrane structure, have not been fully investigated.

The results of the present investigation reveal important changes in the membrane conditions involving the - $\mathrm{SH}$ groups. The observation of a decreased rate of Mal- 6 reaction in the blood of infants during the first hours of life compared to 4-dayold and adult blood, demonstrated the decreased availability of reducing components (including - $\mathrm{SH}$ groups) in the erythrocytes of newborn infants shortly after birth. The lack of differences in the ESR behavior, as well as chemically assayed reacting - $\mathrm{SH}$ groups, between cord blood and 4-day-old washed RBC clearly indicates the role of plasma factors in the reduced amount of reacting $-\mathrm{SH}$ groups and the other reducing components in the red cells during the first hours of life.

The absence of any change of ESR spectra in a system containing Mal- 6 and cell free plasma rules out the possibility that plasma proteins alone could affect the rate of ESR reaction. The finding of no differences between samples with and without platelets and leukocytes also rules out the possibility that changes in cells other than erythrocytes could play a role in the observed changes in ESR behavior occurring from birth to the 4th day of life. Therefore, the present observations strongly suggest that differences in plasma factors between cord blood and blood of infants 4 days old could be responsible for modulating the reaction between Mal- 6 and the reacting -SH groups of RBC. If the plasma factors could induce changes in the state of aggregation of membrane proteins such as spectrin (18), or could modulate the membrane's permeability to oxidative agents remains to be established. Whatever the mechanism may be, the reduced availability of reacting - $\mathrm{SH}$ groups during the first hours of life demonstrates that the susceptibility of RBC to the effect of oxidative agents undergoes important variations within a few hours after birth.

The nature of the plasma factors modulating this behavior remains unknown. A direct influence of plasma and $\mathrm{RBC}$ fatty acid content is unlikely in view of the results of several investigations carried out in newborn infants and animals that demonstrate that an adult erythrocyte fatty acid pattern is far from being reached after 4 days of life (13). On the contrary, other factors such as the well-known variations of plasma hormones cannot be ruled out.

Inasmuch as the observed peculiarity of fetal erythrocytes is related to the membrane structure, it is possible that other cell membranes could be involved in these variations immediately after birth.
Acknowledgments. The authors are grateful to Drs. A. Casini and L. Ciccoli (Institute of General Pathology, University of Siena) for advice and technical assistance in the biochemical methods.

\section{REFERENCES}

1. Oski FA 1982 Disorders of red cell metabolism. In: Oski, FA, Naiman JL (eds) Hematologic Problems in the Newborn, 3rd ed. WB Saunders Company, Philadelphia, pp 97-136

2. Oski FA, Barness LA 1967 Vitamin E deficiency: a previously unrecognized cause of hemolytic anemia in premature infants. J Pediatr 70:211-220

3. Gross RT, Bracci R, Rudolph N, Schroeder E, Kochen J 1967 Hydrogen peroxide toxicity and detoxification in the erythrocytes of newborn infants. Blood 29:481-493

4. Rotilio G, Rigo A, Bracci R, Bagnoli F, Sargentini I, Brunori M 1977 Determination of red blood cell superoxide-dismutase and glutathione peroxidase in newborns in relation to neonatal hemolysis. Clin Chim Acta 81:131-134

5. Pryor WA 1982 Free radical biology: xenobiotics, cancer and aging. Ann NY Acad Sci 393:1-22

6. Foster MA 1981 Electron spin resonance in hematological research. $\mathrm{Br} J$ Haematol 47:1-6

7. Butterfield DA 1982 Spin labeling in disease. In: Berliner LJ, Reuben J (eds) Biological Magnetic Resonance, vol 4. Plenum Press, New York, pp 1-78

8. Butterfield DA, Roses AD, Appel SH, Chestnut DB 1976 Electron spin resonance studies of membrane proteins in erythrocytes in myotonic muscular dystrophy. Arch Biochem Biophys 177:226-234

9. Martini G, Bobbi S, Marconi G, Morini R, Albanese B, Pasquini G, Manescalchi PG, Bartoli V 1984 Studies of erythrocyte membranes by ESR of spin probes. Clin Haemorheol 4:299-309

10. Fung LW-M 1983 Analysis of spin labeled erythrocyte membranes. Ann NY Acad Sci 414:162-168

11. Gaffney BJ 1976 The chemistry of spin labels. In: Berliner LJ (ed) Spin Labeling. Theory and Application, vol 1. Academic Press, New York, pp 183-249

12. Morriset JD, Drott HR 1969 Oxidation of the sulphydryl and disulfide groups by the nitroxide radical. J Biol Chem 244:5083-5084

13. Beutler E 1971 Reduced glutathione (GSH). In: Beutler E (ed) Red Cell Metabolism. A Manual of Biochemical Methods, 1st ed. Grune \& Stratton, New York, pp 103-105

14. Sedlak J, Lindsay RH 1968 Estimation of total, protein bound, and nonprotein sulphydryl groups in tissue with Ellman's reagent. Anal Biochem 25:192205

15. Buonocore G, Berti D, Cito G, Hayek Y, Bracci R 1983 Moderately increased hemolysis in newborn infants with hyperbilirubinemia of unknown etiology. Biol Neonate 44:251-256

16. Ciccoli L, Hayek Y, Berti D, Bracci R 1981 Fatty acid pattern of the erythrocyte lipids and plasma vitamin $E$ in the first days of life. Biol Neonate 40:187195

17. Travis SF, Kumar SP, Delivoria-Papadopoulos M 1981 Red cell metabolic alterations in postnatal life in term infants: glycolytic intermediates and adenosine triphosphate. Pediatr Res 15:34-37

18. Bracci R, Buonocore G, Talluri B, Berni S. Neonatal hyperbilirubinemia. Evidence for a role of the erythrocyte enzyme activities involved in the detoxification of oxygen radicals. Acta Paediatr Scand 77:349-356

19. Farmer BT II, Harman TM, Butterfield DA 1985 ESR studies of the erythrocyte membrane skeletal protein network: influence of the state of aggregation of spectrin on the physical state of membrane proteins, bilayer lipids, and cell surface carbohydrates. Biochim Biophys Acta 821:420-430 